\title{
Partial Fairness in Secure Two-Party Computation
}

\author{
S. Dov Gordon* Jonathan Katz*
}

\begin{abstract}
A seminal result of Cleve (STOC '86) is that, in general, complete fairness is impossible to achieve in two-party computation. In light of this, various techniques for obtaining partial fairness have been suggested in the literature. We propose a definition of partial fairness within the standard real-/ideal-world paradigm that addresses deficiencies of prior definitions. We also show broad feasibility results with respect to our definition: partial fairness is possible for any (randomized) functionality $f: X \times Y \rightarrow Z^{1} \times Z^{2}$ at least one of whose domains or ranges is polynomial in size. Our protocols are always private, and when one of the domains has polynomial size our protocols also simultaneously achieve the usual notion of security with abort. In contrast to some prior work, we rely on standard assumptions only.

We also show that, as far as general feasibility is concerned, our results are optimal. Specifically, there exist functions with super-polynomial domains and ranges for which it is impossible to achieve our definition.
\end{abstract}

\section{Introduction}

In the setting of secure two-party computation, two parties run a protocol that enables each of them to learn a (possibly different) function of their inputs while preserving security properties such as privacy, correctness, input independence, etc. These requirements, and more, are traditionally formalized by comparing a real-world execution of the protocol to an ideal world where there is a trusted entity who performs the computation on behalf of the parties. Informally, a protocol is "secure" if for any real-world adversary $\mathcal{A}$ there is a corresponding ideal-world adversary $\mathcal{S}$ (corrupting the same party) such that an execution of the protocol in the real world with $\mathcal{A}$ is computationally indistinguishable from computing the function in the ideal world with $\mathcal{S}$.

One desirable security property is fairness which, intuitively, ensures that either both parties learn the output or else neither party does. In a "true" ideal world - and this is the ideal world used in the multi-party setting when a majority of parties are honest - fairness is ensured since the trusted party evaluating the function provides output to both parties. Unfortunately, Cleve [11] shows that complete fairness is impossible to achieve, in general, in the two-party setting. For this reason, the usual treatment of secure two-party computation (see [19]) weakens the ideal world to one in which fairness is not guaranteed at all. A protocol is said to be "secure-with-abort" if it can be simulated (as described above) with respect to this less-satisfying ideal world. (Formal definitions of all these notions are given in Appendix A.1.)

Various methods for achieving partial fairness have been suggested; we provide an extensive discussion in Section 1.1. With the exception of [18], however, all previous work has departed from

${ }^{*}$ Dept. of Computer Science, University of Maryland. Email: \{gordon,jkatz\}@cs.umd.edu. Research supported by NSF CAREER award \#0447075 and NSF-CCF \#0830464. 
the traditional real-/ideal-world paradigm in defining partial fairness. (Indeed, addressing this deficiency is explicitly mentioned as an open problem by Goldreich [19, Section 7.7.1.1].) Furthermore, many previously suggested approaches to partial fairness only apply in specific settings (e.g., fair exchange of signatures) or under certain assumptions on the parties' inputs and auxiliary information (e.g., that inputs are chosen uniformly at random), but do not give a "general-purpose" solution that can be used for arbitrary functions computed on arbitrary inputs. Finally, much previous work on partial fairness requires strong cryptographic assumptions, e.g., regarding the precise amount of time needed to solve some problem (even using parallelism).

As discussed, the most desirable (but, in the two-party setting, typically unachievable) definition of security requires computational indistinguishability between the real world and a "true" ideal world where both parties receive output. The usual relaxation of security-with-abort [19] leaves unchanged the requirement of computational indistinguishability, but weakens the ideal world so that fairness is no longer guaranteed at all. Motivated by [25], we suggest an alternate relaxation: keep the ideal world unchanged, but relax the notion of simulation and require instead that the real and ideal worlds be distinguishable with probability at most $\frac{1}{p}+$ negl, where $p$ is some specified polynomial $^{1}$ (see Definition 1 ). We refer to a protocol satisfying this definition as being " $\frac{1}{p}$-secure". Cleve [11] and Moran et al. [29] show $\frac{1}{p}$-secure protocols for two-party coin tossing (where parties have no inputs), but we are not aware of any other results satisfying our definition. In particular, none of the prior approaches for achieving partial fairness yield protocols that are $\frac{1}{p}$-secure.

We propose the notion of $\frac{1}{p}$-security as a new way to approach the problem of partial fairness. We also demonstrate protocols that achieve this definition for a broad class of functionalities. Specifically, let $f_{n}: X_{n} \times Y_{n} \rightarrow Z_{n}^{1} \times Z_{n}^{2}$ be a (randomized) functionality where player 1 (resp., player 2) provides input $x \in X_{n}$ (resp., $y \in Y_{n}$ ) and receives output $z^{1} \in Z_{n}^{1}$ (resp., $z^{2} \in Z_{n}^{2}$ ). (Throughout this paper, $n$ denotes the security parameter. We also impose some technical assumptions on $X_{n}, Y_{n}, Z_{n}^{1}, Z_{n}^{2}$ that are described in Section 2.) For arbitrary polynomial $p$, we show $\frac{1}{p}$-secure protocols for computing $f_{n}$ as long as at least one of $X_{n}, Y_{n}, Z_{n}^{1}, Z_{n}^{2}$ is polynomial size (in $n$ ). Our protocols are always private, and when either $X_{n}$ or $Y_{n}$ is polynomial-size we also achieve the usual notion of security-with-abort. (Relevant definitions are in Appendix A.1.) We assume only the existence of enhanced trapdoor permutations or, more generally, oblivious transfer.

We also prove that our feasibility results are, in general, optimal. First, we demonstrate a deterministic, boolean function $f_{n}: X_{n} \times Y_{n} \rightarrow\{0,1\}$, where $X_{n}$ and $Y_{n}$ both have super-polynomial size, for which no protocol computing $f_{n}$ can simultaneously achieve both security-with-abort and $\frac{1}{p}$-security (for $p>4$ ). We also show a deterministic function $f_{n}: X_{n} \times Y_{n} \rightarrow Z_{n}$, with each of $X_{n}, Y_{n}, Z_{n}$ super-polynomial in size, such that $f_{n}$ cannot be $\frac{1}{p}$-securely computed for $p>2$.

\subsection{Prior Work}

There is an extensive literature devoted to the problem of achieving partial fairness when an honest majority is not present, both for the case of specific functionalities like coin tossing [11, 12, 29] and contract signing/exchanging secrets $[6,27,15,5,13,7]$, as well as for the case of general

\footnotetext{
${ }^{1}$ Katz [25] proposed a related notion of " $\frac{1}{p}$-security-with-abort"; that definition, however, continues to compare the real world with the relaxed ideal world and so again guarantees no fairness at all. A similar relaxation, formalized differently and with different motivation (and again giving no fairness), is also used in [1]. Our definition of $\frac{1}{p}$-security is also similar in spirit to (but weaker than) the notion of $\epsilon$-zero knowledge [14] and is analogous to some definitions of password-based key exchange [20] (although there $p$ is fixed by the size of the password dictionary).
} 
functionalities [31, 17, 3, 21, 16, 30, 18]. Prior work (with the exception of [18]), however, does not consider a simulation-based definition within the standard real/ideal world paradigm as we do here. ${ }^{2}$ Moreover, to the best of our knowledge none of the previous approaches (with the exception of $[11,29]$, that deal only with coin tossing) can be proven $\frac{1}{p}$-secure. Beyond the theoretical advantages of achieving a simulation-based notion of security, our protocols offer several concrete benefits with respect to prior solutions; these are explained in what follows. (See also [23] for an exhaustive discussion.)

One approach that has been suggested [6, 15] for achieving partial fairness is to construct a protocol where, roughly speaking, at every round both parties can recover their output using a "similar" amount of work. This idea was used for general secure computation in $[17,13,7,30]$, and was formalized by Garay et al. [18] within the framework of universal composability [10]. An unsatisfying feature of this approach, no matter how it is implemented, is that the decision of whether an honest party should invest the necessary work and recover the output is not specified as part of the protocol, but is somehow decided "externally". Note that if the adversary knows how this decision is made, then it can abort at "exactly the right time" and violate fairness completely. In this approach there may also be no a priori polynomial bound on the honest party's running time. This approach also seems problematic in defending against an adversary who runs in polynomial time, but has more computational power than honest parties are able to invest. Finally, this technique appears inherently to require strong assumptions regarding the precise time required to solve some specific computational problem.

A second approach, suggested for contract signing/exchanging secrets [27, 5] as well as for computation of general functions [3, 21] (see also [19]), gradually increases both parties' "confidence" in the output. By way of example (and simplifying slightly for the purposes of this discussion), consider the case where both parties receive the same boolean output $z=f(x, y)$ and imagine a protocol running for poly $(n)$ iterations where, in each iteration $i$, the parties learn $b_{i}=z \oplus r_{i}$ with the $\left\{r_{i}\right\}$ independent and $\operatorname{Pr}\left[r_{i}=0\right]=\frac{1}{2}+1 / \operatorname{poly}(n)$. (This is basically the protocol of [3].) When the protocol ends, or when one party aborts, the honest party outputs the majority of the $\left\{b_{i}\right\}$ seen thus far. Protocols of this sort do not appear to apply (at least not without significant changes) to multi-bit outputs or when parties are to receive different outputs. More problematic is the fact that an adversary can significantly bias the output of the honest party. For example, in the protocol just described, an adversary who always aborts after the first iteration causes the honest party to output an almost-random bit. We may contrast this with the standard simulation-based definition where such bias should, in general, be impossible.

Complete fairness. Gordon et al. [24] recently showed that complete fairness is possible in the two-party setting for certain specific functions. Work continuing that direction is complementary to our work here: while we do not yet have a complete characterization of what can be computed with complete fairness, we know that there certainly do exist some functions that cannot be computed with complete fairness [11] and so some relaxation must be considered (at least for some functions). Our feasibility results here apply to a much richer class of functions.

Other work has looked at achieving complete fairness using off-line trusted third parties (e.g., [8]) or in non-standard communication models (e.g., [26]). We work in the standard communication model, and without relying on any trusted parties.

\footnotetext{
${ }^{2}$ Damgård [13] defines a simulation-based notion of partial fairness, but not within the standard framework.
} 


\subsection{Overview of our Approach}

We now give an informal description of our feasibility results (details are in Section 3 ). Let $x$ denote the input of $P_{1}$, let $y$ denote the input of $P_{2}$, and let $f: X \times Y \rightarrow Z$ denote the function they are trying to compute. (For simplicity, here we omit the dependence of $X, Y$, and $Z$ on $n$, and focus on the case where each party receives the same output.) As in [25, 24, 29], our protocols will be composed of two stages, where the first stage can be viewed as a "pre-processing" step and the second stage takes place in a sequence of $r=r(n)$ iterations. The stages have the following form:

\section{First stage.}

1. A value $i^{*} \in\{1, \ldots, r\}$ is chosen according to some distribution (see below). This represents the iteration in which both parties will learn the "true output".

2. Values $a_{1}, \ldots, a_{r}$ and $b_{1}, \ldots, b_{r}$ are generated. For $i<i^{*}$, the $\left\{a_{i}\right\}$ (resp., $\left.\left\{b_{i}\right\}\right)$ are chosen (independently) according to some distribution that is independent of $y$ (resp., $x$ ); see below for further discussion. For $i \geq i^{*}$, however, it holds that $a_{i}=b_{i}=f(x, y)$.

3. Each $a_{i}$ is randomly shared as $a_{i}^{(1)}, a_{i}^{(2)}$ with $a_{i}^{(1)} \oplus a_{i}^{(2)}=a_{i}$ (and similarly for each $b_{i}$ ). The stage concludes with $P_{1}$ being given $a_{1}^{(1)}, b_{1}^{(1)}, \ldots, a_{r}^{(1)}, b_{r}^{(1)}$, and $P_{2}$ being given $a_{1}^{(2)}, b_{1}^{(2)}, \ldots$, $a_{r}^{(2)}, b_{r}^{(2)}$. (Shares are also authenticated with an information-theoretic MAC.)

After this stage, each party has a set of random shares that reveal nothing about the other party's input. This stage can thus be carried out by any protocol that is secure-with-abort (since even a party who aborts after receiving his output in this stage does not learn anything about $f(x, y)$ ).

Second stage. In each iteration $i$, for $i=1, \ldots, r$, the parties do the following: First, $P_{2}$ sends

$a_{i}^{(2)}$ to $P_{1}$ who reconstructs $a_{i}$; then $P_{1}$ sends $b_{i}^{(1)}$ to $P_{2}$ who reconstructs $b_{i}$. (Parties also verify validity of the MAC but we omit this here.) If a party (say, $P_{1}$ ) aborts in some iteration $i$, then the other party (here, $P_{2}$ ) outputs the value reconstructed in the previous iteration (i.e., $b_{i-1}$ ). Otherwise, after reaching iteration $r$ the parties output $a_{r}$ and $b_{r}$, respectively.

To fully specify the protocol we must specify the distribution of $i^{*}$ as well as the distribution of the $a_{i}, b_{i}$ for $i<i^{*}$. As in [25,29], we choose $i^{*}$ uniformly from $\{1, \ldots, r\}$. (In [24] a geometric distribution was used. That would work here, but with slightly worse round complexity.) When $X$ and $Y$ (the domains of $f$ ) are polynomial size, we follow [24] and set $a_{i}=f(x, \hat{y})$ for $\hat{y}$ chosen uniformly from $Y$, and set $b_{i}=f(\hat{x}, y)$ for $\hat{x}$ chosen uniformly (and independently) from $X$. Note that $a_{i}$ (resp., $b_{i}$ ) is independent of $y$ (resp., $x$ ), as desired.

Intuitively, this is partially fair because fairness is violated only if $P_{1}$ aborts exactly in iteration $i^{*}$. (If $P_{1}$ aborts before iteration $i^{*}$ then neither party learns the "correct" value $z=f(x, y$ ), while if it aborts after iteration $i^{*}$ then both parties learn the correct value. An abort by $P_{2}$ in iteration $i^{*}$ does not violate fairness, since by then $P_{1}$ has already learned the output.) We show that even if $P_{1}$ knows the value of $z$ (which it may, depending on partial information $P_{1}$ has about $y$ ), it cannot determine with certainty when iteration $i^{*}$ occurs. Specifically, we prove a general result (see Lemma 2) implying (roughly) that as long as $\operatorname{Pr}\left[a_{i}=z\right] \geq \alpha$ for all $i<i^{*}$, then $P_{1}$ cannot abort in iteration $i^{*}$ except with probability at most $1 / \alpha r$ (recall that $r$ is the number of iterations in the second phase). Since $\operatorname{Pr}\left[a_{i}=f(x, y)\right]=\operatorname{Pr}_{\hat{y} \in Y}[f(x, \hat{y})=f(x, y)] \geq \operatorname{Pr}_{\hat{y} \in Y}[\hat{y}=y]=1 /|Y|$ for any $x, y$, we conclude that setting $r=p \cdot|Y|$, so that $1 / \alpha r=1 / p$, suffices to achieve $\frac{1}{p}$-security. (A small change to the protocol ensures that it is also secure-with-abort; see Section 3.2 for details.) 
We thus get a protocol with polynomially many rounds as long as $Y$ is polynomial size. The same argument, with the parties' roles interchanged, yields a protocol when $X$ is polynomial size.

The above does not work directly when $Y$ has super-polynomial size. To fix this, we must ensure that for every possible $z \in Z$ (the range of $f$ ) we have that $\operatorname{Pr}\left[a_{i}=z\right]$ is noticeable. We do this by changing the distribution of $a_{i}$ (for $i<i^{*}$ ) as follows: with probability $1-1 / q$ choose $a_{i}$ as above, but with probability $1 / q$ choose $a_{i}$ uniformly from $Z$. Now, for any $x, y$, we have $\operatorname{Pr}\left[a_{i}=f(x, y)\right] \geq \frac{1}{q} \cdot \operatorname{Pr}_{a_{i} \in Z}\left[a_{i}=f(x, y)\right] \geq 1 / q|Z|$ and so setting $r=p q|Z|$ ensures that $P_{1}$ cannot abort in iteration $i^{*}$ except with probability at most $1 / p$.

Changing the distribution of $a_{i}$, however, introduces a new problem: if $P_{2}$ aborts prior to iteration $i^{*}$, the output of the honest $P_{1}$ in the real world cannot necessarily be simulated in the ideal world. We show, however, that it can be simulated to within statistical difference $\mathcal{O}(1 / q)$. Taking $q=p$ (along with $r=p q|Z|$ ) thus gives a $\frac{1}{p}$-secure protocol with polynomially many rounds.

\section{Definitions}

Preliminaries. A function $\mu(\cdot)$ is negligible if for every positive polynomial $p(\cdot)$ and all sufficiently large $n$ it holds that $\mu(n)<1 / p(n)$. A distribution ensemble $X=\{X(a, n)\}_{a \in \mathcal{D}_{n}, n \in \mathbb{N}}$ is an infinite sequence of random variables indexed by $a \in \mathcal{D}_{n}$ and $n \in \mathbb{N}$, where $\mathcal{D}_{n}$ may depend on $n$.

For a fixed function $p$, the ensembles $X=\{X(a, n)\}_{a \in \mathcal{D}_{n}, n \in \mathbb{N}}$ and $Y=\{Y(a, n)\}_{a \in \mathcal{D}_{n}, n \in \mathbb{N}}$ are computationally $\frac{1}{p}$-indistinguishable, denoted $X \stackrel{1 / p}{\approx} Y$, if for every non-uniform polynomial-time algorithm $D$ there exists a negligible function $\mu(\cdot)$ such that for every $n$ and every $a \in \mathcal{D}_{n}$

$$
|\operatorname{Pr}[D(X(a, n))=1]-\operatorname{Pr}[D(Y(a, n))=1]| \leq \frac{1}{p(n)}+\mu(n) .
$$

Two distribution ensembles are computationally indistinguishable, denoted $X \stackrel{\mathrm{c}}{\equiv} Y$, if for every $c \in \mathbb{N}$ they are computationally $\frac{1}{n^{c}}$-indistinguishable.

Functionalities. A functionality $\mathcal{F}=\left\{f_{n}\right\}_{n \in \mathbb{N}}$ is a sequence of poly-time computable, randomized mappings $f_{n}: X_{n} \times Y_{n} \rightarrow Z_{n}^{1} \times Z_{n}^{2}$, where $X_{n}$ and $Z_{n}^{1}$ (resp., $Y_{n}$ and $Z_{n}^{2}$ ) denote the input and output of the first (resp., second) party. Our results assume that $X_{n}=\{0,1\}^{\ell(n)}$ for some (known) length parameter $\ell(n)$ (and similarly for $Y_{n}, Z_{n}^{1}$, and $Z_{n}^{2}$ ); however, this can be relaxed to require only that (1) membership in $X_{n}, Y_{n}$ is efficiently decidable and (2) the uniform distribution over $X_{n}, Y_{n}, Z_{n}^{1}, Z_{n}^{2}$ is efficiently sampleable.

We write $f_{n}=\left(f_{n}^{1}, f_{n}^{2}\right)$ if we wish to emphasize the two outputs of $f_{n}$, but stress that if $f_{n}^{1}$ and $f_{n}^{2}$ are randomized then the outputs of $f_{n}^{1}$ and $f_{n}^{2}$ are correlated random variables. If $\operatorname{Pr}\left[f_{n}^{1}(x, y)=f_{n}^{2}(x, y)\right]=1$ for all $x, y$, then we call $f_{n}$ a single-output functionality and write it as $f_{n}: X_{n} \times Y_{n} \rightarrow Z_{n}$. If $\mathcal{F}$ is deterministic, we sometimes call it a function. For notational convenience, we sometimes drop the explicit dependence on $n$.

Two-party computation. A two-party protocol for computing a functionality $\mathcal{F}=\left\{\left(f^{1}, f^{2}\right)\right\}$ is a protocol running in polynomial time and satisfying the following correctness requirement: if party $P_{1}$ begins by holding $1^{n}$ and input $x \in X$, and party $P_{2}$ holds $1^{n}$ and input $y \in Y$, then the joint distribution of the outputs of the parties is statistically close to $\left(f^{1}(x, y), f^{2}(x, y)\right)$.

Security of protocols. We consider static corruptions by active adversaries, who may deviate from the protocol in an arbitrary manner. We define security via the standard real/ideal 
paradigm [19] (based on [28, 2, 9]), where security of a protocol is analyzed by comparing what an adversary can do in a real protocol execution to what it can do in an ideal world that is secure by definition. For completeness, we include in Appendix A.1 descriptions of the standard ideal- and real-world models. There, we define $\operatorname{IDEAL}_{\mathcal{F}, \mathcal{A} \text { (aux) }}(x, y, n)$ as the random variable consisting of the output of the adversary $\mathcal{A}$ and the output of the honest party following a computation of $\mathcal{F}$ in the ideal model (where complete fairness is guaranteed), with security parameter $n$ and parties holding initial inputs $x$ and $y$, respectively, and auxiliary input aux. We also define $\operatorname{REAL}_{\Pi, \mathcal{A}(\text { aux })}(x, y, n)$ as the analogous random variable for the real-world execution of protocol $\Pi$.

Having defined the ideal and real models, we now state our new notion of security. Loosely speaking, our definition asserts that a secure protocol (in the real model) emulates the ideal model (in which a trusted party exists) to within a difference of $\frac{1}{p}$. This is formulated as follows:

Definition 1 Let $\mathcal{F}, \Pi$ be as above, and fix a function p. Protocol $\Pi$ is said to $\frac{1}{p}$-securely compute $\mathcal{F}$ if for every non-uniform probabilistic polynomial-time adversary $\mathcal{A}$ in the real model, there exists a non-uniform probabilistic polynomial-time adversary $\mathcal{S}$ in the ideal model such that

$$
\left\{\operatorname{IDEAL}_{\mathcal{F}, \mathcal{S}(\text { aux })}(x, y, n)\right\}_{(x, y) \in X \times Y, \text { aux } \in\{0,1\}^{*}, n \in \mathbb{N}} \stackrel{1 / p}{\approx}\left\{\operatorname{REAL}_{\Pi, \mathcal{A}(\text { aux })}(x, y, n)\right\}_{(x, y) \in X \times Y, \text { aux } \in\{0,1\}^{*}, n \in \mathbb{N}} .
$$

Although our definition of $\frac{1}{p}$-security allows privacy to be violated with probability $\frac{1}{p}$, in fact all our protocols are fully private (see Definition 14 in Appendix A.1).

\section{$3 \quad \frac{1}{p}$-Secure Computation of General Functionalities}

We begin in Section 3.1 by stating a lemma that forms an essential piece of our analysis in the two sections that follow. In Section 3.2 we demonstrate a private and $\frac{1}{p}$-secure protocol for functionalities defined on polynomial-size domains. A slight modification of this protocol is also simultaneously secure-with-abort. To keep the exposition as simple as possible, we restrict our attention there to single-output functionalities (though the techniques extend easily to the general case). In Section 3.3 we show how to adapt the protocol for functionalities defined over domains of superpolynomial size (but polynomial range), and also generalize to functionalities generating different outputs for each party.

\subsection{A Useful Lemma}

We analyze an abstract game $\Gamma$ between a challenger and an (unbounded) adversary $\mathcal{A}$. The game is parameterized by a value $\alpha \in(0,1]$ and an integer $r \geq 1$. Fix arbitrary distributions $D_{1}, D_{2}$ such that for every $z$ it holds that

$$
\operatorname{Pr}_{a \leftarrow D_{1}}[a=z] \geq \alpha \cdot \operatorname{Pr}_{a \leftarrow D_{2}}[a=z] .
$$

The game $\Gamma(\alpha, r)$ proceeds as follows:

1. The challenger chooses $i^{*}$ uniformly from $\{1, \ldots, r\}$, and then chooses $a_{1}, \ldots, a_{r}$ as follows:

- For $i<i^{*}$, it chooses $a_{i} \leftarrow D_{1}$.

- For $i \geq i^{*}$, it chooses $a_{i} \leftarrow D_{2}$. 
2. The challenger and $\mathcal{A}$ then interact in a sequence of at most $r$ iterations. In iteration $i$ :

- The challenger gives $a_{i}$ to the adversary.

- The adversary can either abort or continue. In the former case, the game stops. In the latter case, the game continues to the next iteration.

3. $\mathcal{A}$ wins if it aborts the game in iteration $i^{*}$.

Let $\operatorname{Win}(\alpha, r)$ denote the maximum probability with which $\mathcal{A}$ wins the above game.

Lemma 2 For any $D_{1}, D_{2}$ satisfying Equation (1), it holds that $\operatorname{Win}(\alpha, r) \leq 1 / \alpha r$.

Proof: Fix $D_{1}, D_{2}$ satisfying (1). We prove the lemma by induction on $r$. When $r=1$ the lemma is trivially true; for completeness, we also directly analyze the case $r=2$. Since $\mathcal{A}$ is unbounded we may assume it is deterministic. So without loss of generality, we may assume the adversary's strategy is determined by a set $S$ in the support of $D_{2}$ such that $\mathcal{A}$ aborts in the first iteration iff $a_{1} \in S$, and otherwise aborts in the second iteration (no matter what). We have

$$
\begin{aligned}
\operatorname{Pr}[\mathcal{A} \text { wins }] & =\operatorname{Pr}\left[\mathcal{A} \text { wins and } i^{*}=1\right]+\operatorname{Pr}\left[\mathcal{A} \text { wins and } i^{*}=2\right] \\
& =\frac{1}{2} \cdot \operatorname{Pr}_{a \leftarrow D_{2}}[a \in S]+\frac{1}{2} \cdot\left(1-\operatorname{Pr}_{a \leftarrow D_{1}}[a \in S]\right) \\
& \leq \frac{1}{2} \cdot \operatorname{Pr}_{a \leftarrow D_{2}}[a \in S]+\frac{1}{2} \cdot\left(1-\alpha \cdot \operatorname{Pr}_{a \leftarrow D_{2}}[a \in S]\right) \\
& =\frac{1}{2}+\frac{1}{2} \cdot\left((1-\alpha) \cdot \operatorname{Pr}_{a \leftarrow D_{2}}[a \in S]\right) \leq 1-\alpha / 2,
\end{aligned}
$$

where the first inequality is due to Equation (1). One can easily verify that $1-\alpha / 2 \leq 1 / 2 \alpha$ when $\alpha>0$. We have thus proved $\operatorname{Win}(\alpha, 2) \leq 1 / 2 \alpha$.

Assume $\operatorname{Win}(\alpha, r) \leq 1 / \alpha r$, and we now bound $\operatorname{Win}(\alpha, r+1)$. As above, let $S$ denote a set in the support of $D_{2}$ such that $\mathcal{A}$ aborts in the first iteration iff $a_{1} \in S$. If $\mathcal{A}$ does not abort in the first iteration, and the game does not end, then the conditional distribution of $i^{*}$ is uniform in $\{2, \ldots, r+1\}$ and the game $\Gamma(\alpha, r+1)$ from this point forward is exactly equivalent to the game $\Gamma(\alpha, r)$. In particular, conditioned on the game $\Gamma(\alpha, r+1)$ not ending after the first iteration, the best strategy for $\mathcal{A}$ is to play whatever is the best strategy in game $\Gamma(\alpha, r)$. We thus have

$$
\begin{aligned}
\operatorname{Win}(\alpha, r+1) & =\operatorname{Pr}\left[\mathcal{A} \text { wins and } i^{*}=1\right]+\operatorname{Pr}\left[\mathcal{A} \text { wins and } i^{*}>1\right] \\
& =\frac{1}{r+1} \cdot \operatorname{Pr}_{a \leftarrow D_{2}}[a \in S]+\frac{r}{r+1} \cdot\left(1-\operatorname{Pr}_{a \leftarrow D_{1}}[a \in S]\right) \cdot \operatorname{Win}(\alpha, r) \\
& \leq \frac{1}{r+1} \cdot \operatorname{Pr}_{a \leftarrow D_{2}}[a \in S]+\frac{1}{\alpha(r+1)} \cdot\left(1-\alpha \cdot \operatorname{Pr}_{a \leftarrow D_{2}}[a \in S]\right) . \\
& =\frac{1}{\alpha(r+1)} .
\end{aligned}
$$

This completes the proof. 


\section{ShareGen $_{r}$}

Inputs: The security parameter is $n$. Let the inputs to ShareGen be $\in X_{n}$ and $y \in Y_{n}$. (If one of the received inputs is not in the correct domain, a default input is substituted.)

\section{Computation:}

1. Define values $a_{1}, \ldots, a_{r}$ and $b_{1}, \ldots, b_{r}$ in the following way:

- Choose $i^{*}$ uniformly at random from $\{1, \ldots, r\}$.

- For $i=1$ to $i^{*}-1$ do:

- Choose $\hat{y} \leftarrow Y_{n}$ and set $a_{i}=f_{n}(x, \hat{y})$.

- Choose $\hat{x} \leftarrow X_{n}$ and set $b_{i}=f_{n}(\hat{x}, y)$.

- Compute $z=f_{n}(x, y)$. For $i=i^{*}$ to $r$, set $a_{i}=b_{i}=z$.

2. For $1 \leq i \leq r$, choose $\left(a_{i}^{(1)}, a_{i}^{(2)}\right)$ and $\left(b_{i}^{(1)}, b_{i}^{(2)}\right)$ as random secret sharings of $a_{i}$ and $b_{i}$, respectively. (I.e., $a_{i}^{(1)}$ is random and $a_{i}^{(1)} \oplus a_{i}^{(2)}=a_{i}$.)

3. Compute $k_{a}, k_{b} \leftarrow \operatorname{Gen}\left(1^{n}\right)$. For $1 \leq i \leq r$, let $t_{i}^{a}=\operatorname{Mac}_{k_{a}}\left(i \| a_{i}^{(2)}\right)$ and $t_{i}^{b}=\operatorname{Mac}_{k_{b}}\left(i \| b_{i}^{(1)}\right)$.

\section{Output:}

1. Send to $P_{1}$ the values $a_{1}^{(1)}, \ldots, a_{r}^{(1)}$ and $\left(b_{1}^{(1)}, t_{1}^{b}\right), \ldots,\left(b_{r}^{(1)}, t_{r}^{b}\right)$, and the MAC-key $k_{a}$.

2. Send to $P_{2}$ the values $\left(a_{1}^{(2)}, t_{1}^{a}\right), \ldots,\left(a_{r}^{(2)}, t_{r}^{a}\right)$ and $b_{1}^{(2)}, \ldots, b_{r}^{(2)}$, and the MAC-key $k_{b}$.

Figure 1: Functionality ShareGen .

\section{$3.2 \frac{1}{\mathrm{p}}$-Security for Functionalities with Polynomial-Size Domain}

In this section, we describe a protocol that works for functionalities where at least one of the domains is polynomial-size. (We stress that the protocol works directly for randomized functionalities; the standard reduction from randomized to deterministic functionalities [19] would not apply here since, in general, it makes the domain too large.) Although a small modification of the protocol works even when the parties receive different outputs, for simplicity we assume here that the parties compute a single-output function. We return to the more general setting in the following section.

Theorem 3 Let $\mathcal{F}=\left\{f_{n}: X_{n} \times Y_{n} \rightarrow Z_{n}\right\}$ be a (randomized) functionality where $\left|Y_{n}\right|=$ poly $(n)$. Assuming the existence of enhanced trapdoor permutations, for any polynomial $p$ there is an $\mathcal{O}\left(p \cdot\left|Y_{n}\right|\right)$-round protocol computing $\mathcal{F}$ that is private and $\frac{1}{p}$-secure.

Proof: As described in Section 1.2, our protocol $\Pi$ consists of two stages. Let $p$ be a polynomial, and set $r=p \cdot\left|Y_{n}\right|$. We implement the first stage of $\Pi$ using a sub-protocol $\pi$ for computing a

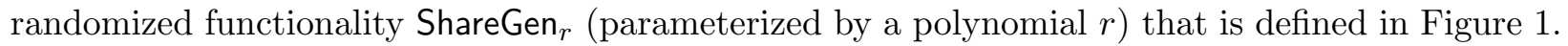
This functionality returns shares to each party, authenticated using an information-theoretically secure $r$-time MAC (Gen, Mac, Vrfy). (See Appendix A.2 for a definition.) In the second stage of $\Pi$ the parties exchange these shares in a sequence of $r$ iterations as described in Figure 2.

We analyze our protocol in a hybrid model where there is a trusted party computing ShareGen $r$ according to the second ideal model where a malicious $P_{1}$ can abort the trusted party before it sends output to the honest party. We prove privacy and $\frac{1}{p}$-security of $\Pi$ in this hybrid model; it follows as in [9] that if we use a sub-protocol for computing ShareGen $r$ that is secure-with-abort, then the real-world protocol $\Pi$ is private and $\frac{1}{p}$-secure. 


\section{Protocol 1}

Inputs: Party $P_{1}$ has input $x$ and party $P_{2}$ has input $y$. The security parameter is $n$. Let $r=p \cdot\left|Y_{n}\right|$. The protocol:

\section{Preliminary phase:}

(a) $P_{1}$ chooses $\hat{y} \in Y_{n}$ uniformly at random, and sets $a_{0}=f_{n}(x, \hat{y})$. Similarly, $P_{2}$ chooses $\hat{x} \in X_{n}$ uniformly at random, and sets $b_{0}=f_{n}(\hat{x}, y)$.

(b) Parties $P_{1}$ and $P_{2}$ run a protocol $\pi$ to compute ShareGen ${ }_{r}$, using their inputs $x$ and $y$.

(c) If $P_{2}$ receives $\perp$ from the above computation, it outputs $b_{0}$ and halts. Otherwise, the parties proceed to the next step.

(d) Denote the output of $P_{1}$ from $\pi$ by $a_{1}^{(1)}, \ldots, a_{r}^{(1)},\left(b_{1}^{(1)}, t_{1}^{b}\right), \ldots,\left(b_{r}^{(1)}, t_{r}^{b}\right)$, and $k_{a}$.

(e) Denote the output of $P_{2}$ from $\pi$ by $\left(a_{1}^{(2)}, t_{1}^{a}\right), \ldots,\left(a_{r}^{(2)}, t_{r}^{a}\right), b_{1}^{(2)}, \ldots, b_{r}^{(2)}$, and $k_{b}$.

2. For $i=1, \ldots, r$ do:

$P_{2}$ sends the next share to $P_{1}$ :

(a) $P_{2}$ sends $\left(a_{i}^{(2)}, t_{i}^{a}\right)$ to $P_{1}$.

(b) $P_{1}$ receives $\left(a_{i}^{(2)}, t_{i}^{a}\right)$ from $P_{2}$. If $\operatorname{Vrfy}_{k_{a}}\left(i \| a_{i}^{(2)}, t_{i}^{a}\right)=0$ (or if $P_{1}$ received an invalid message, or no message), then $P_{1}$ outputs $a_{i-1}$ and halts.

(c) If $\operatorname{Vrfy}_{k_{a}}\left(i \| a_{i}^{(2)}, t_{i}^{a}\right)=1$, then $P_{1}$ sets $a_{i}=a_{i}^{(1)} \oplus a_{i}^{(2)}$ (and continues running the protocol).

$P_{1}$ sends the next share to $P_{2}$ :

(a) $P_{1}$ sends $\left(b_{i}^{(1)}, t_{i}^{b}\right)$ to $P_{2}$.

(b) $P_{2}$ receives $\left(b_{i}^{(1)}, t_{i}^{b}\right)$ from $P_{1}$. If $\mathrm{Vrfy}_{k_{b}}\left(i \| b_{i}^{(1)}, t_{i}^{b}\right)=0$ (or if $P_{2}$ received an invalid message, or no message), then $P_{2}$ outputs $b_{i-1}$ and halts.

(c) If $\operatorname{Vrfy}_{k_{b}}\left(i \| b_{i}^{(1)}, t_{i}^{b}\right)=1$, then $P_{2}$ sets $b_{i}=b_{i}^{(1)} \oplus b_{i}^{(2)}$ (and continues running the protocol).

3. If all $r$ iterations have been run, party $P_{1}$ outputs $a_{r}$ and party $P_{2}$ outputs $b_{r}$.

Figure 2: Generic protocol for computing a functionality $f_{n}$.

We first consider the case of a malicious $P_{1}$. Intuition for the following claim was given in Section 1.2. The formal statement and proof follow.

Claim 4 Let $\Pi^{\text {hy }}$ denote an execution of $\Pi$ in a hybrid model with access to an ideal functionality computing ShareGen $_{r}$ (with abort). For every non-uniform, polynomial-time adversary $\mathcal{A}$ corrupting $P_{1}$ and running $\Pi^{\text {hy }}$, there exists a non-uniform, polynomial-time adversary $\mathcal{S}$ corrupting $P_{1}$ and running in the ideal world with access to an ideal functionality computing $\mathcal{F}$ (with complete fairness), such that $\frac{1}{p}$-security holds, i.e.,

$$
\left\{\operatorname{IDEAL}_{\mathcal{F}, \mathcal{S}(\text { aux })}(x, y, n)\right\}_{x \in X_{n}, y \in Y_{n}, \text { aux } \in\{0,1\}^{*}} \stackrel{1 / p}{\approx}\left\{\operatorname{HYBRID}_{\Pi^{\text {hy }}, \mathcal{A}(\text { aux })}(x, y, n)\right\}_{x \in X_{n}, y \in Y_{n}, \text { aux } \in\{0,1\}^{*}},
$$

and privacy holds, i.e.,

$$
\left\{\operatorname{OUT}_{\mathcal{F}, \mathcal{S}(\text { aux })}^{\mathcal{S}}(x, y, n)\right\}_{x \in X_{n}, y \in Y_{n}, \text { aux } \in\{0,1\}^{*}} \equiv\left\{\operatorname{VIEW}_{\Pi^{\text {hy }}, \mathcal{A}(\text { aux })}^{\mathcal{A}}(x, y, n)\right\}_{x \in X_{n}, y \in Y_{n}, \text { aux } \in\{0,1\}^{*}} .
$$


Proof: We construct a simulator $\mathcal{S}$ that is given black-box access to $\mathcal{A}$. For readability in what follows, we ignore the $M A C$-tags and keys. That is, we do not mention the fact that $\mathcal{S}$ computes MAC-tags for messages it sends to $\mathcal{A}$, nor do we mention the fact that $\mathcal{S}$ must verify the MAC-tags on the messages sent by $\mathcal{A}$. When we say that $\mathcal{A}$ "aborts", we include in this the event that $\mathcal{A}$ sends an invalid message, or a message whose tag does not pass verification. We also drop the subscript $n$ from our notation and write $X, Y$ in place of $X_{n}, Y_{n}$.

1. $\mathcal{S}$ invokes $\mathcal{A}$ on the input ${ }^{3} x^{\prime}$, the auxiliary input, and the security parameter $n$. The simulator also chooses $\hat{x} \in X$ uniformly at random (it will send $\hat{x}$ to the trusted party, if needed).

2. $\mathcal{S}$ receives the input $x$ of $\mathcal{A}$ to the computation of the functionality ShareGen ${ }_{r}$. (If $x \notin X$ a default input is substituted.)

3. $\mathcal{S}$ sets $r=p \cdot|Y|$, and chooses uniformly distributed shares $a_{1}^{(1)}, \ldots, a_{r}^{(1)}$ and $b_{1}^{(1)}, \ldots, b_{r}^{(1)}$. Then, $\mathcal{S}$ gives these shares to $\mathcal{A}$ as its output from the computation of ShareGen ${ }_{r}$.

4. If $\mathcal{A}$ sends abort to the trusted party computing $\operatorname{ShareGen}_{r}$, then $\mathcal{S}$ sends $\hat{x}$ to the trusted party computing $f$, outputs whatever $\mathcal{A}$ outputs, and halts. Otherwise (i.e., if $\mathcal{A}$ sends continue), $\mathcal{S}$ proceeds as below.

5. Choose $i^{*}$ uniformly from $\{1, \ldots, r\}$.

6. For $i=1$ to $i^{*}-1$ :

(a) $\mathcal{S}$ chooses $\hat{y} \in Y$ uniformly at random, computes $a_{i}=f(x, \hat{y})$, and sets $a_{i}^{(2)}=a_{i}^{(1)} \oplus a_{i}$. It gives $a_{i}^{(2)}$ to $\mathcal{A}$. (A fresh $\hat{y}$ is chosen in every iteration.)

(b) If $\mathcal{A}$ aborts, then $\mathcal{S}$ sends $\hat{x}$ to the trusted party, outputs whatever $\mathcal{A}$ outputs, and halts.

7. For $i=i^{*}$ to $r$ :

(a) If $i=i^{*}$ then $\mathcal{S}$ sends $x$ to the trusted party computing $f$ and receives $z=f(x, y)$.

(b) $\mathcal{S}$ sets $a_{i}^{(2)}=a_{i}^{(1)} \oplus z$ and gives $a_{i}^{(2)}$ to $\mathcal{A}$.

(c) If $\mathcal{A}$ aborts, then $\mathcal{S}$ outputs whatever $\mathcal{A}$ outputs, and halts. If $\mathcal{A}$ does not abort, then $\mathcal{S}$ proceeds.

8. If $\mathcal{A}$ never aborted (and all $r$ iterations are done), $\mathcal{S}$ outputs what $\mathcal{A}$ outputs and halts.

It is immediate that the view of $\mathcal{A}$ in the simulation above is distributed identically to its view in $\Pi^{\text {hy }}$; privacy follows. We now prove $\frac{1}{p}$-security.

Ignoring the possibility of a MAC forgery, we claim that the statistical difference between an execution of $\mathcal{A}$, running $\Pi$ in a hybrid world with access to an ideal functionality computing ShareGen ${ }_{r}$, and an execution of $\mathcal{S}$, running in an ideal world with access to an ideal functionality computing $f$, is at most $1 / p$. (Thus, taking into account the possibility of a MAC forgery makes the statistical difference at most $1 / p+\mu(n)$ for some negligible function $\mu$.) To see this, let $y$ denote the input of the honest $P_{2}$ and consider three cases depending on when the adversary aborts:

\footnotetext{
${ }^{3}$ We reserve $x$ for the value input by $\mathcal{A}$ to the computation of ShareGen ${ }_{r}$.
} 
1. $\mathcal{A}$ aborts in round $i<i^{*}$. Conditioned on this event, the view of $\mathcal{A}$ is identically distributed in the two worlds (and is independent of $y$ ), and the output of the honest party is $f(\hat{x}, y)$ for $\hat{x}$ chosen uniformly in $X$.

2. $\mathcal{A}$ aborts in round $i>i^{*}$ (or never). Conditioned on this, the view of $\mathcal{A}$ is again distributed identically in the two worlds, and in both worlds the output of the honest party is $f(x, y)$.

3. $\mathcal{A}$ aborts in round $i=i^{*}$ : here, although the view of $\mathcal{A}$ is still identical in both worlds, the output of the honest party is not: in the hybrid world the honest party will output $f(\hat{x}, y)$, for $\hat{x}$ chosen uniformly in $X$, while in the ideal world the honest party will output $f(x, y)$.

However, Lemma 2 implies that this event occurs with probability at most $1 / p$. To see this, let $D_{1}$ denote the distribution of $a_{i}$ for $i<i^{*}$ (i.e., this is the distribution defined by the output of $f(x, \hat{y})$, for $\hat{y}$ chosen uniformly from $Y$ ), and let $D_{2}$ denote the distribution of $a_{i^{*}}$ (i.e., the distribution defined by the output of $f(x, y)$ ). For any $z \in Z$ we have

$$
\begin{aligned}
\operatorname{Pr}_{a \leftarrow D_{1}}[a=z] & \stackrel{\text { def }}{=} \operatorname{Pr}_{\hat{y} \leftarrow Y}[f(x, \hat{y})=z] \\
& \geq \frac{1}{|Y|} \cdot \operatorname{Pr}[f(x, y)=z]=\frac{1}{|Y|} \cdot \operatorname{Pr}_{a \leftarrow D_{2}}[a=z]
\end{aligned}
$$

Taking $\alpha=1 /|Y|$ and applying Lemma 2 , we see that $\mathcal{A}$ aborts in iteration $i^{*}$ with probability at most $1 / \alpha r=|Y| /|Y| p=1 / p$.

This completes the proof of the claim.

Next we consider the case of a malicious $P_{2}$. A proof of the following is almost identical to that of Claim 4; in fact, the proof is simpler and we can prove a stronger notion of security (namely, where complete fairness holds) since $P_{1}$ always "gets the output first" in every iteration of $\Pi$. For these reasons, a proof is omitted.

Claim 5 (Informal) Let $\Pi^{\text {hy }}$ denote an execution of $\Pi$ in a hybrid model where the parties have access to an ideal functionality computing ShareGen ${ }_{r}$ (with abort). Then for any adversary corrupting $P_{2}$, protocol $\Pi^{\text {hy }}$ securely computes $\mathcal{F}$.

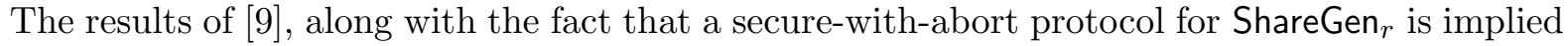
by the existence of enhanced trapdoor permutations, complete the proof of Theorem 3.

Achieving security-with-abort. As written, the protocol is not secure-with-abort. However, the protocol can be modified easily so that it is (without affecting $\frac{1}{p}$-security): $\operatorname{simply}_{\text {have ShareGen }}$ choose $i^{*}$ uniformly from $\{2, \ldots, r+1\}$ and set $b_{i^{*}-1}=\perp$, where $\perp$ is some distinguished value outside the range of $f$. Although this allows a malicious $P_{2}$ to identify exactly when iteration $i^{*}$ occurs, this does not affect security since by that time $P_{1}$ has already received the correct output.

\section{$3.3 \quad \frac{1}{\mathrm{p}}$-Security for Functionalities with Polynomial-Size Range}

The protocol from the previous section does not apply to functions on domains of super-polynomial size, since the round complexity is linear in the size of the smaller domain. Here we show how to extend the protocol to handle arbitrary domains if the range of the function (for at least one of the parties) is polynomial size. We now also explicitly take into account the case when parties obtain different outputs. Intuition for the changes we introduce is given in Section 1.2. 


\section{ShareGen ${ }_{p, r}^{\prime}$}

Inputs: The security parameter is $n$. Let the inputs to ShareGen ${ }_{p, r}^{\prime}$ be $x \in X_{n}$ and $y \in Y_{n}$. (If one of the received inputs is not in the correct domain, a default input is substituted.)

\section{Computation:}

1. Define values $a_{1}, \ldots, a_{r}$ and $b_{1}, \ldots, b_{r}$ in the following way:

- Choose $i^{*}$ uniformly at random from $\{1, \ldots, r\}$.

- For $i=1$ to $i^{*}-1$ do:

- Choose $\hat{x} \leftarrow X_{n}$ and set $b_{i}=f_{n}^{2}(\hat{x}, y)$.

- With probability $\frac{1}{p}$, choose $z \leftarrow Z_{n}^{1}$ and set $a_{i}=z$. With the remaining probability $1-\frac{1}{p}$, choose $\hat{y} \leftarrow Y$ and set $a_{i}=f_{n}^{1}(x, \hat{y})$.

- Compute $z_{1}=f_{n}^{1}(x, y)$ and $z_{2}=f_{n}^{2}(x, y)$ (if $f_{n}=\left(f_{n}^{1}, f_{n}^{2}\right)$ is randomized, these values are computed using the same random tape). For $i=i^{*}$ to $r$, set $a_{i}=z_{1}$ and $b_{i}=z_{2}$.

2. For $1 \leq i \leq r$, choose $\left(a_{i}^{(1)}, a_{i}^{(2)}\right)$ and $\left(b_{i}^{(1)}, b_{i}^{(2)}\right)$ as random secret sharings of $a_{i}$ and $b_{i}$, respectively. (E.g., $a_{i}^{(1)}$ is random and $a_{i}^{(1)} \oplus a_{i}^{(2)}=a_{i}$.)

3. Compute $k_{a}, k_{b} \leftarrow \operatorname{Gen}\left(1^{n}\right)$. For $1 \leq i \leq r$, let $t_{i}^{a}=\operatorname{Mac}_{k_{a}}\left(i \| a_{i}^{(2)}\right)$ and $t_{i}^{b}=\operatorname{Mac}_{k_{b}}\left(i \| b_{i}^{(1)}\right)$.

\section{Output:}

1. Send to $P_{1}$ the values $a_{1}^{(1)}, \ldots, a_{r}^{(1)}$ and $\left(b_{1}^{(1)}, t_{1}^{b}\right), \ldots,\left(b_{r}^{(1)}, t_{r}^{b}\right)$, and the MAC-key $k_{a}$.

2. Send to $P_{2}$ the values $\left(a_{1}^{(2)}, t_{1}^{a}\right), \ldots,\left(a_{r}^{(2)}, t_{r}^{a}\right)$ and $b_{1}^{(2)}, \ldots, b_{r}^{(2)}$, and the MAC-key $k_{b}$.

Figure 3: Functionality ShareGen ${ }_{p, r}^{\prime}$.

Theorem 6 Let $\mathcal{F}=\left\{f_{n}: X_{n} \times Y_{n} \rightarrow Z_{n}^{1} \times Z_{n}^{2}\right\}$ be a (randomized) functionality, where $\left|Z_{n}^{1}\right|=$ poly $(n)$. Assuming the existence of enhanced trapdoor permutations, for any polynomial $p$ there is an $\mathcal{O}\left(p^{2} \cdot\left|Z_{n}^{1}\right|\right)$-round protocol computing $\mathcal{F}$ that is private and $\frac{1}{p}$-secure.

Proof: Our protocol $\Pi$ is, once again, composed of two stages. The second stage is identical to the second stage of the previous protocol (see Figure 2), except that the number of iterations $r$ is now set to $r=p^{2} \cdot\left|Z_{n}^{1}\right|$. The first stage generates shares using a sub-routine $\pi$ computing a different functionality ShareGen ${ }_{p, r}$, parameterized by both $p$ and $r$ and described in Figure 3.

We again analyze our protocol in a hybrid model, where there is now a trusted party computing ShareGen ${ }_{p, r}^{\prime}$. (Once again, $P_{1}$ can abort the computation of ShareGen ${ }_{p, r}^{\prime}$ in the ideal world.) We prove privacy and $\frac{1}{p}$-security of $\Pi$ in this hybrid model, implying [9] that if the parties use a secure-with-abort protocol for computing ShareGen ${ }_{p, r}^{\prime}$, then the real-world protocol $\Pi$ is private and $\frac{1}{p}$-secure. We first consider the case of a malicious $P_{1}$.

Claim 7 Let $\Pi^{\text {hy }}$ denote an execution of $\Pi$ in a hybrid model with access to an ideal functionality computing ShareGen ${ }_{p, r}^{\prime}$ (with abort). For every non-uniform, polynomial-time adversary $\mathcal{A}$ corrupting $P_{1}$ and running $\Pi^{\text {hy }}$, there exists a non-uniform, polynomial-time adversary $\mathcal{S}$ corrupting $P_{1}$ and running in the ideal world with access to an ideal functionality computing $\mathcal{F}$ (with complete fairness), such that $\frac{1}{p}$-security holds, i.e.,

$$
\left\{\operatorname{IDEAL}_{\mathcal{F}, \mathcal{S}(\text { aux })}(x, y, n)\right\}_{x \in X_{n}, y \in Y_{n}, \text { aux } \in\{0,1\}^{*}} \stackrel{1 / p}{\approx}\left\{\operatorname{HYBRID}_{\Pi^{\text {hy }}, \mathcal{A}(\text { aux })}(x, y, n)\right\}_{x \in X_{n}, y \in Y_{n}, \text { aux } \in\{0,1\}^{*}},
$$


and privacy holds, i.e.,

$$
\left\{\operatorname{OUT}_{\mathcal{F}, \mathcal{S}(\text { aux })}^{\mathcal{S}}(x, y, n)\right\}_{x \in X_{n}, y \in Y_{n}, \text { aux } \in\{0,1\}^{*}} \equiv\left\{\operatorname{VIEW}_{\Pi^{\text {hy }}, \mathcal{A}(\text { aux })}^{\mathcal{A}}(x, y, n)\right\}_{x \in X_{n}, y \in Y_{n}, \text { aux } \in\{0,1\}^{*}} .
$$

Proof: The simulator used to prove this claim is essentially the same as the simulator used in the proof of Claim 4, except that in step 6(a) the distribution on $a_{i}$ (for $i<i^{*}$ ) is changed to the one used by ShareGen ${ }_{p, r}^{\prime}$. The analysis is similar, too, except for bounding the probability that $\mathcal{A}$ aborts in iteration $i^{*}$. To bound this probability we will again rely on Lemma 2, but now distribution $D_{1}$ (i.e., the distribution of $a_{i}$ for $i<i^{*}$ ) is different. Let $y$ denote the input of $P_{2}$. Note that, by construction of ShareGen ${ }_{p, r}^{\prime}$, for any $z \in Z_{n}^{1}$ we have $\operatorname{Pr}_{a \leftarrow D_{1}}[a=z] \geq \frac{1}{p} \cdot \frac{1}{\left|Z_{n}^{1}\right|}$. Regardless of $f^{1}$ and $y$, it therefore holds for all $z \in Z_{n}^{1}$ that

$$
\operatorname{Pr}_{a \leftarrow D_{1}}[a=z] \geq \frac{1}{p \cdot\left|Z_{n}^{1}\right|} \cdot \operatorname{Pr}_{a \leftarrow D_{2}}[a=z] .
$$

Setting $\alpha=1 / p \cdot\left|Z_{n}^{1}\right|$ and applying Lemma 2 , we see that $\mathcal{A}$ aborts in iteration $i^{*}$ with probability at most

$$
\frac{1}{\alpha r}=\frac{p \cdot\left|Z_{n}^{1}\right|}{p^{2} \cdot\left|Z_{n}^{1}\right|}=\frac{1}{p}
$$

This completes the proof of the claim.

We next consider the case of a malicious $P_{2}$. Note that, in contrast to Claim 5, here we claim only $\frac{1}{p}$-security (and privacy).

Claim 8 Let $\Pi^{\text {hy }}$ denote an execution of $\Pi$ in a hybrid model with access to an ideal functionality computing ShareGen ${ }_{p, r}^{\prime}$ (with abort). For every non-uniform, polynomial-time adversary $\mathcal{A}$ corrupting $P_{2}$ and running $\Pi^{\text {hy }}$, there exists a non-uniform, polynomial-time adversary $\mathcal{S}$ corrupting $P_{2}$ and running in the ideal world with access to an ideal functionality computing $\mathcal{F}$ (with complete fairness), such that $\frac{1}{p}$-security holds, i.e.,

$$
\left\{\operatorname{IDEAL}_{\mathcal{F}, \mathcal{S}(\text { aux })}(x, y, n)\right\}_{x \in X_{n}, y \in Y_{n}, \text { aux } \in\{0,1\}^{*}} \stackrel{1 / p}{\approx}\left\{\operatorname{HYBRID}_{\Pi^{\text {hy }}, \mathcal{A}(\text { aux })}(x, y, n)\right\}_{x \in X_{n}, y \in Y_{n}, \text { aux } \in\{0,1\}^{*}},
$$

and privacy holds, i.e.,

$$
\left\{\operatorname{OUT}_{\mathcal{F}, \mathcal{S}(\text { aux })}^{\mathcal{S}}(x, y, n)\right\}_{x \in X_{n}, y \in Y_{n}, \text { aux } \in\{0,1\}^{*}} \equiv\left\{\operatorname{VIEW}_{\Pi^{\text {hy }}, \mathcal{A}(\text { aux })}^{\mathcal{A}}(x, y, n)\right\}_{x \in X_{n}, y \in Y_{n}, \text { aux } \in\{0,1\}^{*}} .
$$

Proof: The simulator $\mathcal{S}$ in this case is fairly obvious, but we include it for completeness. Once again, for readability we ignore the presence of the MAC-tags and keys.

1. $\mathcal{S}$ invokes $\mathcal{A}$ on the input $y^{\prime}$, the auxiliary input, and the security parameter $n$. The simulator also chooses $\hat{y} \in Y$ uniformly at random (it will send $\hat{y}$ to the trusted party, if needed).

2. $\mathcal{S}$ receives the input $y$ of $\mathcal{A}$ to the computation of the functionality ShareGen ${ }_{p, r}^{\prime}$. (If $y \notin Y$ a default input is substituted.)

3. $\mathcal{S}$ sets $r=p^{2} \cdot\left|Z^{1}\right|$, and chooses uniformly distributed shares $a_{1}^{(1)}, \ldots, a_{r}^{(1)}$ and $b_{1}^{(1)}, \ldots, b_{r}^{(1)}$. Then, $\mathcal{S}$ gives these shares to $\mathcal{A}$ as its output from the computation of ShareGen ${ }_{p, r}^{\prime}$. 
4. Choose $i^{*}$ uniformly from $\{1, \ldots, r\}$.

5. For $i=1$ to $i^{*}-1$ :

(a) $\mathcal{S}$ chooses $\hat{x} \in X$ uniformly at random, computes $b_{i}=f^{2}(\hat{x}, y)$, and sets $b_{i}^{(1)}=b_{i}^{(2)} \oplus b_{i}$. It gives $b_{i}^{(1)}$ to $\mathcal{A}$. (Note that a fresh $\hat{x}$ is chosen in every iteration.)

(b) If $\mathcal{A}$ aborts, then $\mathcal{S}$ sends $\hat{y}$ to the trusted party, outputs whatever $\mathcal{A}$ outputs, and halts.

6. For $i=i^{*}$ to $r$ :

(a) If $i=i^{*}$ then $\mathcal{S}$ sends $y$ to the trusted party computing $f$ and receives $z=f^{2}(x, y)$.

(b) $\mathcal{S}$ sets $b_{i}^{(1)}=b_{i}^{(2)} \oplus z$ and gives $b_{i}^{(1)}$ to $\mathcal{A}$.

(c) If $\mathcal{A}$ aborts, then $\mathcal{S}$ outputs whatever $\mathcal{A}$ outputs, and halts. If $\mathcal{A}$ does not abort, then $\mathcal{S}$ proceeds.

7. If $\mathcal{A}$ has never aborted (and all $r$ iterations are done), then $\mathcal{S}$ outputs whatever $\mathcal{A}$ outputs and halts.

Privacy is immediate, and so we focus on $\frac{1}{p}$-security. Ignoring the possibility of a MAC forgery, we claim that the statistical difference between an execution of $\mathcal{A}$, running $\Pi$ in a hybrid world with access to an ideal functionality computing ShareGen ${ }_{p, r}^{\prime}$, and an execution of $\mathcal{S}$, running in an ideal world with access to an ideal functionality computing $\mathcal{F}$, is at most $1 / p$. (Thus, taking into account the possibility of a MAC forgery makes the statistical difference at most $1 / p+\mu(n)$ for some negligible function $\mu$.) The view of $\mathcal{A}$ is identical in the two worlds; the only issue is the output of the honest $P_{1}$ holding input $x$. Specifically, if $\mathcal{A}$ aborts in any iteration prior to $i^{*}$ then, in the ideal-world interaction with $\mathcal{S}$, party $P_{1}$ outputs $f^{1}(x, \hat{y})$ for a uniformly chosen $\hat{y} \in Y$. In the hybrid world, however, the output of $P_{1}$ is given by the distribution of $a_{i}$ (for $i<i^{*}$ ) as determined by ShareGen ${ }_{p, r}^{\prime}$. However, these two distributions are within statistical difference (at most) $1 / p$. The claim follows.

The results of [9], along with the fact that a secure-with-abort protocol for ShareGen ${ }_{p, r}$ is implied by the existence of enhanced trapdoor permutations, complete the proof of Theorem 6 .

\section{Optimality of Our Results}

We show that the results of the previous section are optimal as far as generic feasibility is concerned.

\subsection{Impossibility of $\frac{1}{p}$-Security and Security-with-Abort Simultaneously}

In Section 3.2 (cf. the remark at the end of that section) we showed a protocol achieving $\frac{1}{p}$-security and security-with-abort simultaneously for functionalities where at least one of the domains is polynomial-size. We show that if both domains are super-polynomial in size then, in general, it is impossible to achieve both these criteria at once.

Theorem 9 Let $\mathcal{F}=\left\{\mathrm{EQ}_{n}:\{0,1\}^{\ell(n)} \times\{0,1\}^{\ell(n)} \rightarrow\{0,1\}\right\}$, where $\mathrm{EQ}_{n}$ denotes the equality function on strings and $\ell(n)=\omega(\log n)$. Let $\Pi$ be any protocol computing $\mathcal{F}$. If $\Pi$ is secure-with-abort, then $\Pi$ does not $\frac{1}{p}$-securely compute $\mathcal{F}$ for any $p \geq 4+\frac{1}{\operatorname{poly}(n)}$. 
Proof: Let $\Pi$ be a protocol that computes $\mathcal{F}$ and is secure-with-abort. Assume without loss of generality that $P_{2}$ sends the first message in $\Pi$ and that $P_{1}$ sends the last message. Say $\Pi$ has $r=r(n)$ iterations for some polynomial $r$, where an iteration consists of a message sent by $P_{2}$ followed by a message sent by $P_{1}$. Let $a_{0}$ denote the value that $P_{1}$ outputs if $P_{2}$ sends nothing, and let $a_{i}$, for $1 \leq i \leq r$, denote the value that $P_{1}$ outputs if $P_{2}$ aborts after sending its iteration- $i$ message. Similarly, let $b_{0}$ denote the value that $P_{2}$ outputs if $P_{1}$ sends nothing, and let $b_{i}$, for $1 \leq i \leq r$, denote the value that $P_{2}$ outputs if $P_{1}$ aborts after sending its iteration- $i$ message. We may assume without loss of generality that, for all $i$, we have $a_{i} \in\{0,1\}$ and $b_{i} \in\{0,1, \perp\}$.

We will consider two experiments involving an execution of $\Pi$. In the first, $x$ and $y$ are chosen uniformly and independently from $\{0,1\}^{\ell(n)}$; the parties are given inputs $x$ and $y$, respectively; and the parties then run protocol $\Pi$ honestly. We denote the probability of events in this experiment by $\operatorname{Pr}_{\text {rand }}[\cdot]$. In the second experiment, $x$ is chosen uniformly from $\{0,1\}^{\ell(n)}$ and $y$ is set equal to $x$; these inputs are given to the parties and they run the protocol honestly as before. We denote the probability of events in this probability space by $\operatorname{Pr}_{\text {eq }}[\cdot]$.

Claim $10 \operatorname{Pr}_{\text {rand }}\left[a_{0}=1 \vee \cdots \vee a_{r}=1\right]$ and $\operatorname{Pr}_{\text {rand }}\left[b_{0}=1 \vee \cdots \vee b_{r}=1\right]$ are negligible.

Proof: This follows from the fact that $\Pi$ is secure-with-abort. If, say, it were the case that $\operatorname{Pr}_{\text {rand }}\left[a_{0}=1 \vee \cdots \vee a_{r}=1\right]$ is not negligible, then we could consider an adversarial $P_{2}$ that runs the protocol honestly but aborts at a random round. This would cause the honest $P_{1}$ to output 1 with non-negligible probability in the real world, whereas $P_{1}$ outputs 1 with only negligible probability in the ideal world (since the parties are given independent, random inputs).

Assume for simplicity that $\Pi$ has perfect correctness, i.e., that $a_{r}=b_{r}=\mathrm{EQ}(x, y)$ when the two parties run the protocol honestly holding initial inputs $x$ and $y$. (This assumption is not necessary, but allows us to avoid having to deal with annoying technicalities.) Then

$$
\operatorname{Pr}_{\text {eq }}\left[a_{0}=1 \vee \cdots \vee a_{r}=1\right]=\operatorname{Pr}_{\text {eq }}\left[b_{0}=1 \vee \cdots \vee b_{r}=1\right]=1
$$

since, in particular, $\operatorname{Pr}_{\text {eq }}\left[a_{r}=1\right]=\operatorname{Pr}_{\text {eq }}\left[b_{r}=1\right]=1$. In a given execution, let $i^{*}$ denote the lowest index for which $a_{i^{*}}=1$, and let $j^{*}$ denote the lowest index for which $b_{j^{*}}=1$. Since

$$
\operatorname{Pr}_{\mathrm{eq}}\left[i^{*} \leq j^{*}\right]+\operatorname{Pr}_{\mathrm{eq}}\left[i^{*}>j^{*}\right]=1
$$

at least one of the terms on the left-hand side is at least $1 / 2$. We assume that $\operatorname{Pr}_{\text {eq }}\left[i^{*} \leq j^{*}\right] \geq 1 / 2$, but the same argument (swapping the roles of the parties) applies if $\operatorname{Pr}_{\text {eq }}\left[i^{*}>j^{*}\right] \geq 1 / 2$.

Consider now a third experiment that is a mixture of the previous two. Specifically, in this experiment a random bit $b$ is chosen; if $b=0$ then the parties are given inputs $x$ and $y$ as in the first experiment (i.e., chosen uniformly and independently at random), while if $b=1$ then the parties are given (random) $x=y$ as in the second experiment. The parties then run protocol $\Pi$ honestly. We denote the probability of events in this probability space by $\operatorname{Pr}_{3}^{\text {real }}[\cdot]$. We use the superscript real to distinguish this from an ideal-world version of this experiment where the bit $b$ is chosen uniformly and the parties are given $x$ and $y$ generated accordingly, but now the parties interact with an ideal party computing EQ without abort (i.e., in the first ideal model). We denote the probability of events in this experiment by $\operatorname{Pr}_{3}^{\text {ideal }}[\cdot]$.

Consider an execution of the third experiment (in either the real or ideal worlds), in the case when $P_{1}$ is malicious. Let guess denote the event that $P_{1}$ correctly guesses the value of the bit $b$, 
and let out 2 denote the output of $P_{2}$. It is not hard to show that

$$
\operatorname{Pr}_{3}^{\text {ideal }}\left[\text { guess } \wedge \text { out }_{2} \neq 1\right] \leq \frac{1}{2}
$$

(Note that out ${ }_{2} \in\{0,1\}$ in the first ideal world.) Now take the following real-world adversary $\mathcal{A}$ corrupting $P_{1}$ : upon receiving input $x$, adversary $\mathcal{A}$ runs $\Pi$ honestly but computes $a_{i}$ after receiving each iteration- $i$ message from $P_{2}$. Then:

- If, at some point, $a_{i}=1$ then $\mathcal{A}$ aborts the protocol (before sending the iteration- $i$ message on behalf of $\left.P_{1}\right)$ and outputs the guess " $b=1$ ".

- If $a_{i}=0$ for all $i$, then $\mathcal{A}$ simply runs the protocol to the end (including the final message of the protocol) and outputs the guess " $b=0$ ".

We have:

$$
\begin{aligned}
& \operatorname{Pr}_{3}^{\text {real }}[\text { guess } \wedge \text { out } \\
&\neq 1] \\
&\left.=\frac{1}{2} \cdot \operatorname{Pr}_{\text {rand }}\left[\text { guess } \wedge \text { out }_{2} \neq 1\right]+\frac{1}{2} \cdot \operatorname{Pr}_{\text {eq }} \text { [guess } \wedge \text { out }_{2} \neq 1\right] \\
& \geq \frac{1}{2} \cdot \operatorname{Pr}_{\text {rand }}\left[a_{1}=0 \wedge \cdots \wedge a_{r}=0 \wedge b_{r}=0\right]+\frac{1}{2} \cdot \operatorname{Pr}_{\text {eq }}\left[i^{*} \leq j^{*}\right] \\
& \geq \frac{1}{2} \cdot(1-\operatorname{negl}(n))+\frac{1}{4}=\frac{3}{4}-\operatorname{negl}(n),
\end{aligned}
$$

using Claim 10 for the second inequality. Equations (2) and (3) show that $\Pi$ cannot also be $\frac{1}{p}$-secure for any $p \geq 4+\frac{1}{\operatorname{poly}(n)}$.

\subsection{Impossibility of $\frac{1}{p}$-Security for General Functions}

Our results show that $\frac{1}{p}$-security is achievable for any functionality $f: X_{n} \times Y_{n} \rightarrow Z_{n}^{1} \times Z_{n}^{2}$ if at least one of $X_{n}, Y_{n}, Z_{n}^{1}, Z_{n}^{2}$ has polynomial size. Here, we demonstrate that this limitation is inherent.

Define a deterministic, single-output function $\mathcal{F}=\left\{\mathrm{Swap}_{n}\right\}$ with

$$
\mathrm{Swap}_{n}:\{0,1\}^{\omega(\log n)} \times\{0,1\}^{\omega(\log n)} \rightarrow\{0,1\}^{\omega(\log n)}
$$

as follows: Fix some $\ell(n)=\omega(\log n)$. Let (Gen, Mac, Vrfy) denote an information-theoretic, one-time MAC for messages of length $2 \cdot \ell(n)$ with key length $\mathcal{O}(\ell(n))$ and tag length $\ell(n)$.

$$
\begin{aligned}
& \operatorname{Swap}_{n}\left(\left(x_{1}, t_{1}, k_{2}, k_{2}^{\prime}, t_{2}^{\prime}\right),\left(x_{2}, t_{2}, k_{1}, k_{1}^{\prime}, t_{1}^{\prime}\right)\right) \\
& \stackrel{\text { def }}{=}\left\{\begin{array}{cl}
\left(x_{1}, x_{2}\right) & \text { if } \operatorname{Vrfy}_{k_{1}}\left(x_{1}, t_{1}\right)=\operatorname{Vrfy}_{k_{2}}\left(x_{2}, t_{2}\right)=1 \\
\perp & \text { otherwise }
\end{array} .\right.
\end{aligned}
$$

(Both parties receive the same output $\left(x_{1}, x_{2}\right)$ in the first case. Note that the functionality ignores the final two inputs of the parties; those values will be used in the proof, below.)

Theorem 11 Function $\mathcal{F}$ cannot be $\frac{1}{p}$-securely computed for any $p \geq 2+\frac{1}{\operatorname{poly}(n)}$.

Proof: Consider an ideal-world computation of Swap where: 
- $x_{1}, x_{2}$ are chosen uniformly at random from $\{0,1\}^{2 \ell(n)}$.

- $k_{1}, k_{1}^{\prime}, k_{2}, k_{2}^{\prime}$ are output by Gen $\left(1^{n}\right)$ (i.e., they are random MAC-keys).

- $t_{1}=\operatorname{Mac}_{k_{1}}\left(x_{1}\right), t_{1}^{\prime}=\operatorname{Mac}_{k_{1}^{\prime}}\left(x_{1}\right), t_{2}=\operatorname{Mac}_{k_{2}}\left(x_{2}\right)$, and $t_{2}^{\prime}=\operatorname{Mac}_{k_{2}^{\prime}}\left(x_{2}\right)$.

- $P_{1}$ is given input $\left(x_{1}, t_{1}, k_{2}, k_{2}^{\prime}, t_{2}^{\prime}\right)$

- $P_{2}$ is given input $\left(x_{2}, t_{2}, k_{1}, k_{1}^{\prime}, t_{1}^{\prime}\right)$.

Define a win for $P_{1}$ as the event that $P_{1}$ outputs $x_{2}$ while $P_{2}$ fails to output $x_{1}$. (A win for $P_{2}$ is defined analogously.) It is easy to see that, e.g., a malicious $P_{1}$ cannot win in the ideal world, where complete fairness is guaranteed, except with negligible probability. This is because $x_{2}$ is a uniform $2 \ell(n)$-bit value, while the only information $P_{1}$ has about $x_{2}$ initially is the $\ell(n)$-bit tag $t_{2}^{\prime}$. Thus, the only way for $P_{1}$ to learn $x_{2}$ is to submit to the trusted party some input $\left(\hat{x}_{1}, \hat{t}_{1}, \hat{k}_{2}\right)$ for which $\operatorname{Vrfy}_{k_{1}}\left(\hat{x}_{1}, \hat{t}_{1}\right)=1$; unless $\hat{x}_{1}=x_{1}$, however, this condition holds with negligible probability.

In any real-world computation of Swap, however, there must be one party who "gets its output first" with probability at least $1 / 2$, and can identify exactly when this occurs using the final two components of its input. More formally, say we have an $r$-iteration protocol $\Pi$ computing Swap where $P_{2}$ sends the first message and $P_{1}$ sends the last message. Let $a_{i}$, for $i=0, \ldots, r$, denote the second component of the value $P_{1}$ would output if $P_{2}$ aborts the protocol after sending its iteration- $i$ message, and let $b_{i}$ denote the first component of the value that $P_{2}$ would output if $P_{1}$ aborts the protocol after sending its iteration- $i$ message. Each value $a_{i}$ and $b_{i}$ can be computed in polynomial time after receiving the other party's iteration- $i$ message. We can therefore define an adversary $P_{1}^{*}$ that acts as follows:

Run the protocol honestly until the first round where $\operatorname{Vrfy}_{k_{2}^{\prime}}\left(a_{i}, t_{2}^{\prime}\right)=1$; then output $a_{i}$ and abort.

An adversary $P_{2}^{*}$ can be defined analogously. Note that if, e.g., $\operatorname{Vrfy}_{k_{2}^{\prime}}\left(a_{i}, t_{2}^{\prime}\right)=1$ then $a_{i}=x_{2}$ except with negligible probability; this follows from the information-theoretic security of the MAC along with the fact that the execution of $\Pi$ is independent of $k_{2}^{\prime}, t_{2}^{\prime}$.

Let $i$ denote the first round in which $\operatorname{Vrfy}_{k_{2}^{\prime}}\left(a_{i}, t_{2}^{\prime}\right)=1$, and let $j$ denote the first round in which $\operatorname{Vrfy}_{k_{1}^{\prime}}\left(b_{j}, t_{1}^{\prime}\right)=1$. Assuming for simplicity that $\Pi$ has perfect correctness, we have

$$
\operatorname{Pr}[i \leq j]+\operatorname{Pr}[j>i]=1 .
$$

Further, since $\mid \operatorname{Pr}\left[P_{1}^{*}\right.$ wins $]-\operatorname{Pr}[i \leq j] \mid$ and $\mid \operatorname{Pr}\left[P_{2}^{*}\right.$ wins $]-\operatorname{Pr}[i>j] \mid$ are both negligible, we see that either $P_{1}^{*}$ or $P_{2}^{*}$ wins in the real world with probability at least $1 / 2-\operatorname{negl}(n)$. Since an adversary wins in the ideal world with negligible probability, this rules out $\frac{1}{p}$-security for $p>2$.

Theorem 11 does not contradict any previous work on fair exchange, since those works consider different models (e.g., exchange of unauthenticated values) and/or definitions of partial fairness than we do here. In particular, in some previous work on fair exchange the running time of the honest party is not bounded by a fixed polynomial, whereas in our setting we require this to be the case. 


\section{Conclusions and Open Questions}

Our work offers a clean definition of partial fairness within the standard real/ideal world paradigm, and settles the question of the general feasibility of achieving this notion in the two-party setting. Several compelling questions remain:

- An easy modification of our second impossibility result (cf. Theorem 11) rules out our definition of partial fairness for the interesting special case of exchanging digital signatures. What is the appropriate (simulation-based?) notion of partial fairness for that setting?

- We can show a function $\mathcal{F}=\left\{f_{n}: X_{n} \times Y_{n} \rightarrow Z_{n}\right\}$ for which any protocol computing $\mathcal{F}$ with $\frac{1}{p}$-security requires $\min \left\{p,\left|X_{n}\right|,\left|Y_{n}\right|\right\}$ rounds. This leaves a gap as compared to Theorem 3 .

- The question of partial fairness in the multi-party setting (with dishonest majority) is wide open. We are not aware of any results in this direction except for the case of coin tossing [11, $29,4]$, or functions where complete fairness is possible [22].

\section{Acknowledgments}

The second author would like to thank Yehuda Lindell for many enlightening discussions on this topic, one of which prompted the inclusion of Section 4.1. We also thank the authors of [29] for sharing a copy of their manuscript with us.

\section{References}

[1] Y. Aumann and Y. Lindell. Security against covert adversaries: Efficient protocols for realistic adversaries. In 4th Theory of Cryptography Conference - TCC 2007, volume 4392 of LNCS, pages 137-156. Springer, 2007.

[2] D. Beaver. Foundations of secure interactive computing. In Advances in Cryptology Crypto '91, volume 576 of $L N C S$, pages 377-391. Springer, 1992.

[3] D. Beaver and S. Goldwasser. Multiparty computation with faulty majority. In 30th Annual Symposium on Foundations of Computer Science (FOCS), pages 468-473. IEEE, 1989.

[4] A. Beimel, E. Omri, and I. Orlov. Protocols for multiparty coin toss with dishonest majority. In Advances in Cryptology - Crypto 2010. To appear.

[5] M. Ben-Or, O. Goldreich, S. Micali, and R. Rivest. A fair protocol for signing contracts. IEEE Trans. Information Theory, 36(1):40-46, 1990.

[6] M. Blum. How to exchange (secret) keys. ACM Transactions on Computer Systems, 1:175-193, 1984.

[7] D. Boneh and M. Naor. Timed commitments. In Advances in Cryptology - Crypto 2000, volume 1880 of $L N C S$, pages 236-254. Springer, 2000.

[8] C. Cachin and J. Camenisch. Optimistic fair secure computation. In Advances in Cryptology - Crypto 2000, volume 1880 of LNCS, pages 93-111. Springer, 2000. 
[9] R. Canetti. Security and composition of multiparty cryptographic protocols. Journal of Cryptology, 13(1):143-202, 2000.

[10] R. Canetti. Universally composable security: A new paradigm for cryptographic protocols. In 42nd Annual Symposium on Foundations of Computer Science (FOCS), pages 136-145. IEEE, 2001.

[11] R. Cleve. Limits on the security of coin flips when half the processors are faulty. In 18th Annual ACM Symposium on Theory of Computing (STOC), pages 364-369. ACM Press, 1986.

[12] R. Cleve. Controlled gradual disclosure schemes for random bits and their applications. In Advances in Cryptology - Crypto '89, volume 435 of LNCS, pages 573-588. Springer, 1990.

[13] I. Damgård. Practical and provably secure release of a secret and exchange of signatures. Journal of Cryptology, 8(4):201-222, 1995.

[14] C. Dwork, M. Naor, and A. Sahai. Concurrent zero-knowledge. J. ACM, 51(6):851-898, 2004.

[15] S. Even, O. Goldreich, and A. Lempel. A randomized protocol for signing contracts. Comm. ACM, 28(6):637-647, 1985.

[16] M. Franklin. Complexity and Security of Distributed Protocols. PhD thesis, Columbia University, 1993.

[17] Z. Galil, S. Haber, and M. Yung. Cryptographic computation: Secure faut-tolerant protocols and the public-key model. In Advances in Cryptology - Crypto '87, volume 293 of LNCS, pages 135-155. Springer, 1988.

[18] J. A. Garay, P. D. MacKenzie, M. Prabhakaran, and K. Yang. Resource fairness and composability of cryptographic protocols. In 3rd Theory of Cryptography Conference - TCC 2006, volume 3876 of $L N C S$, pages 404-428. Springer, 2006.

[19] O. Goldreich. Foundations of Cryptography, vol. 2: Basic Applications. Cambridge University Press, Cambridge, UK, 2004.

[20] O. Goldreich and Y. Lindell. Session-key generation using human passwords only. Journal of Cryptology, 19(3):241-340, 2006.

[21] S. Goldwasser and L. A. Levin. Fair computation of general functions in presence of immoral majority. In Advances in Cryptology - Crypto '90, volume 537 of LNCS, pages 77-93. Springer, 1991.

[22] S. Gordon and J. Katz. Complete fairness in multi-party computation without an honest majority. In 6th Theory of Cryptography Conference - TCC 2009, volume 5444 of LNCS, pages 19-35. Springer, 2009.

[23] S. D. Gordon. Fairness in Secure Computation. PhD thesis, University of Maryland, 2010.

[24] S. D. Gordon, C. Hazay, J. Katz, and Y. Lindell. Complete fairness in secure two-party computation. In 40th Annual ACM Symposium on Theory of Computing (STOC), pages 413422. ACM Press, 2008. 
[25] J. Katz. On achieving the "best of both worlds" in secure multiparty computation. In 39th Annual ACM Symposium on Theory of Computing (STOC), pages 11-20. ACM Press, 2007.

[26] M. Lepinski, S. Micali, C. Peikert, and A. Shelat. Completely fair SFE and coalition-safe cheap talk. In 23rd Annual ACM Symposium on Principles of Distributed Computing (PODC), pages 1-10. ACM Press, 2004.

[27] M. Luby, S. Micali, and C. Rackoff. How to simultaneously exchange a secret bit by flipping a symmetrically-biased coin. In 24th Annual Symposium on Foundations of Computer Science (FOCS), pages 23-30. IEEE, 1983.

[28] S. Micali and P. Rogaway. Secure computation. In Advances in Cryptology - Crypto '91, volume 576 of LNCS, pages 392-404. Springer, 1992.

[29] T. Moran, M. Naor, and G. Segev. An optimally fair coin toss. In 6th Theory of Cryptography Conference - TCC 2009, volume 5444 of LNCS, pages 1-18. Springer, 2009.

[30] B. Pinkas. Fair secure two-party computation. In Advances in Cryptology - Eurocrypt 2003, volume 2656 of LNCS, pages 87-105. Springer, 2003.

[31] A. C.-C. Yao. How to generate and exchange secrets. In 27th Annual Symposium on Foundations of Computer Science (FOCS), pages 162-167. IEEE, 1986.

\section{A Additional Definitions}

\section{A.1 Secure Two-Party Computation}

Execution in the real model. We first consider the real model in which a two-party protocol $\Pi$ is executed by $P_{1}$ and $P_{2}$ (and there is no trusted party). In this case, the adversary $\mathcal{A}$ gets the inputs of the corrupted party and some auxiliary input aux and then starts running the protocol, sending all messages on behalf of the corrupted party using an arbitrary polynomial-time strategy. The honest party follows the instructions of $\Pi$.

Let $\Pi$ be a two-party protocol computing $\mathcal{F}$. Let $\mathcal{A}$ be a non-uniform probabilistic polynomialtime machine with auxiliary input aux. We let $\operatorname{vIEW}_{\Pi, \mathcal{A}(\text { aux })}^{\mathcal{A}}(x, y, n)$ be the random variable denoting the entire view of the adversary following an execution of $\Pi$ as described above, and let $\operatorname{OUT}_{\Pi, \mathcal{A}(\text { aux })}^{\text {hon }}(x, y, n)$ be the random variable denoting the output of the honest party. Set

$$
\operatorname{REAL}_{\Pi, \mathcal{A}(\text { (aux) }}(x, y, n) \stackrel{\text { def }}{=}\left(\operatorname{VIEW}_{\Pi, \mathcal{A}(\text { aux })}^{\mathcal{A}}(x, y, n), \operatorname{OUT}_{\Pi, \mathcal{A}(\text { aux })}^{\text {hon }}(x, y, n)\right) .
$$

Execution in the first ideal model. Here we describe the first ideal model, where complete fairness is guaranteed. The parties are $P_{1}$ and $P_{2}$, and there is an adversary $\mathcal{A}$ who has corrupted one of them. An ideal execution for the computation of $\mathcal{F}=\left\{\left(f_{n}^{1}, f_{n}^{2}\right)\right\}$ proceeds as follows:

Inputs: $P_{1}$ and $P_{2}$ hold $1^{n}$ and inputs $x \in X_{n}$ and $y \in Y_{n}$, respectively; the adversary $\mathcal{A}$ receives an auxiliary input aux. 
Send inputs to trusted party: The honest party sends its input to the trusted party. The corrupted party controlled by $\mathcal{A}$ may send any value of its choice. Denote the pair of inputs sent to the trusted party by $\left(x^{\prime}, y^{\prime}\right)$. (We assume that if $x^{\prime} \notin X_{n}$ the trusted party sets $x^{\prime}$ to some default element in $X_{n}$, and likewise if $y^{\prime} \notin Y_{n}$.)

Trusted party sends outputs: The trusted party chooses $r$ uniformly at random and sends $f_{n}^{1}\left(x^{\prime}, y^{\prime} ; r\right)$ to $P_{1}$ and $f_{n}^{2}\left(x^{\prime}, y^{\prime} ; r\right)$ to party $P_{2}$. (Observe that this ensures complete fairness.)

Outputs: The honest party outputs whatever it was sent by the trusted party, and $\mathcal{A}$ outputs an arbitrary (probabilistic polynomial-time computable) function of its view.

We let $\operatorname{OUT}_{\mathcal{F}, \mathcal{A} \text { (aux) }}^{\mathcal{A}}(x, y, n)\left(\right.$ resp., $\left.\operatorname{OUT}_{\mathcal{F}, \mathcal{A}(\text { aux })}^{\text {hon }}(x, y, n)\right)$ be the random variable denoting the output of $\mathcal{A}$ (resp., the honest party) following an execution in the ideal model as described above. Set

$$
\operatorname{IDEAL}_{\mathcal{F}, \mathcal{A}(\text { aux })}(x, y, n) \stackrel{\text { def }}{=}\left(\operatorname{OUT}_{\mathcal{F}, \mathcal{A} \text { (aux) }}^{\mathcal{A}}(x, y, n), \operatorname{OUT}_{\mathcal{F}, \mathcal{A}(\text { aux })}^{\text {hon }}(x, y, n)\right) .
$$

We can now define the strongest notion of security:

Definition 12 Let $\mathcal{F}, \Pi$ be as above. Protocol $\Pi$ is said to securely compute $\mathcal{F}$ if for every nonuniform probabilistic polynomial-time adversary $\mathcal{A}$ in the real model, there exists a non-uniform probabilistic polynomial-time adversary $\mathcal{S}$ in the ideal model such that

$$
\left\{\operatorname{IDEAL}_{\mathcal{F}, \mathcal{S}(\text { aux })}(x, y, n)\right\}_{(x, y) \in X \times Y, \text { aux } \in\{0,1\}^{*}} \stackrel{\mathrm{c}}{\equiv}\left\{\operatorname{REAL}_{\Pi, \mathcal{A}(\text { aux })}(x, y, n)\right\}_{(x, y) \in X \times Y, \text { aux } \in\{0,1\}^{*}} .
$$

(Recall that $X \stackrel{\mathrm{c}}{\equiv} Y$ denotes that the two distribution ensembles $X, Y$ are computationally indistinguishable, in the standard sense.)

Execution in the second ideal model. In this model, fairness is not guaranteed at all. Nevertheless, this is the usual model in which security of 2-party protocols is proved [19, Section 7.2.3] since security in the sense of Definition 12 is, in general, unachievable in the 2-party setting. The second ideal model is identical to the first ideal model, with the exception of the following step:

Trusted party sends outputs: The trusted party chooses $r$ uniformly at random and computes $z_{1}=f_{n}^{1}\left(x^{\prime}, y^{\prime} ; r\right)$ and $z_{2}=f_{n}^{2}\left(x^{\prime}, y^{\prime} ; r\right)$. If $\mathcal{A}$ controls $P_{2}$, the trusted party sends $z_{1}$ to $P_{1}$ and sends $z_{2}$ to $P_{2}$.

If $\mathcal{A}$ controls $P_{1}$, then the trusted party sends $z_{1}$ to $P_{1}$, who then sends either continue or abort to the trusted party. In the first case, the trusted party then sends $z_{2}$ to $P_{2}$. Otherwise, the trusted party sends $\perp$ to $P_{2}$.

We stress that only a malicious $P_{1}$ is given the opportunity to abort the computation.

We let $\operatorname{IDEAL}_{\mathcal{F}, \mathcal{A} \text { (aux) }}^{\perp}(x, y, n)$ be the random variable consisting of the output of the adversary and the output of the honest party following an execution in the second ideal model. Security in this weaker model is defined analogously to Definition 12:

Definition 13 Let $\mathcal{F}, \Pi$ be as above. Protocol $\Pi$ is said to securely compute $\mathcal{F}$ with abort if for every non-uniform probabilistic polynomial-time adversary $\mathcal{A}$ in the real model, there exists a nonuniform probabilistic polynomial-time adversary $\mathcal{S}$ in the ideal model such that

$$
\left\{\operatorname{IDEAL}_{\mathcal{F}_{,} \mathcal{S}(\text { aux })}^{\perp}(x, y, n)\right\}_{(x, y) \in X \times Y, \text { aux } \in\{0,1\}^{*}} \stackrel{\mathrm{c}}{=}\left\{\operatorname{REAL}_{\Pi, \mathcal{A}(\text { aux })}(x, y, n)\right\}_{(x, y) \in X \times Y, \text { aux } \in\{0,1\}^{*}} .
$$


Finally, we define privacy which is weaker than both of the previous definitions. The notion of privacy captures the requirement that the adversary not learn any information about the honest party's input (other than what is implied by the output), but it does not ensure fairness or prevent an adversary from biasing the honest party's output improperly.

Definition 14 Let $\mathcal{F}, \Pi$ be as above. Protocol $\Pi$ is said to privately compute $\mathcal{F}$ if for every nonuniform probabilistic polynomial-time adversary $\mathcal{A}$ in the real model, there exists a non-uniform probabilistic polynomial-time adversary $\mathcal{S}$ in the ideal model such that

$$
\left\{\operatorname{OUT}_{\mathcal{F}, \mathcal{S}(\text { aux })}^{\mathcal{S}}(x, y, n)\right\}_{(x, y) \in X \times Y, \text { aux } \in\{0,1\}^{*}} \stackrel{\stackrel{\mathrm{c}}{=}}{=}\left\{\operatorname{VIEW}_{\Pi, \mathcal{A}(\text { aux })}^{\mathcal{A}}(x, y, n)\right\}_{(x, y) \in X \times Y, \text { aux } \in\{0,1\}^{*}} .
$$

\section{A.2 Information-Theoretic MACs}

We briefly review the standard definition for information-theoretically secure message authentication codes (MACs). A message authentication code consists of three polynomial-time algorithms (Gen, Mac, Vrfy). The key-generation algorithm Gen takes as input the security parameter $1^{n}$ in unary and outputs a key $k$. The message authentication algorithm Mac takes as input a key $k$ and a message $M \in\{0,1\}^{\leq n}$, and outputs a tag $t$; we write this as $t=\operatorname{Mac}_{k}(M)$. The verification algorithm Vrfy takes as input a key $k$, a message $M \in\{0,1\}^{\leq n}$, and a tag $t$, and outputs a bit $b$; we write this as $b=\operatorname{Vrfy}_{k}(M, t)$. We regard $b=1$ as acceptance and $b=0$ as rejection, and require that for all $n$, all $k$ output by $\operatorname{Gen}\left(1^{n}\right)$, all $M \in\{0,1\} \leq n$, it holds that $\operatorname{Vrfy}_{k}\left(M, \operatorname{Mac}_{k}(M)\right)=1$.

We say (Gen, Mac, Vrfy) is a secure $m$-time $M A C$, where $m$ may be a function of $n$, if no computationally unbounded adversary can output a valid tag on a new message after seeing valid tags on $m$ other messages. For our purposes, we do not require security against an adversary who adaptively chooses its $m$ messages for which to obtain a valid tag; it suffices to consider a nonadaptive definition where the $m$ messages are fixed in advance. (Nevertheless, known constructions satisfy the stronger requirement.) Formally:

Definition 15 Message authentication code (Gen, Mac, Vrfy) is an information-theoretically secure $m$-time MAC if for any sequence of messages $M_{1}, \ldots, M_{m}$ and any adversary $\mathcal{A}$, the following is negligible in the security parameter $n$ :

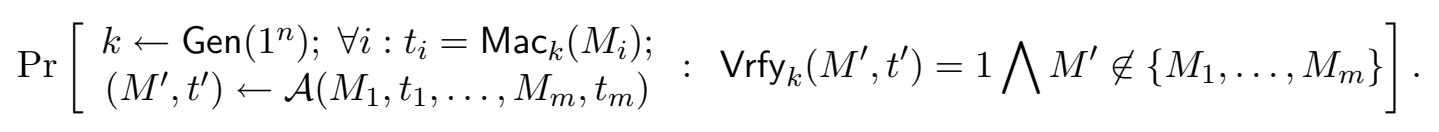

